\begin{tabular}{|c|l|}
\hline Title & Missing modes in the density of states of fractal networks \\
\hline Author(s) & Y akubo, K.; Courtens, E.; Nakayama, T. \\
\hline Citation & $\begin{array}{l}\text { PHY SICAL REVIEW B, 42(1), 1078-1081 } \\
\text { https://doi.org/L0.1103/PhysRevB.42.1078 }\end{array}$ \\
\hline Issue Date & 1990-07-15 \\
\hline Doc URL & http://hdl.handle.net/2115/5655 \\
\hline Rights & Copyright $\odot$ 1990 A merican Physical Society \\
\hline Type & article \\
\hline File Information & PRB42-1.pdf \\
\hline
\end{tabular}

Instructions for use 


\title{
Missing modes in the density of states of fractal networks
}

\author{
K. Yakubo \\ Department of Applied Physics, Hokkaido University, Sapporo 060, Japan \\ E. Courtens \\ IBM Research Division, Zürich Research Laboratory, CH-8803 Rüschlikon, Switzerland \\ T. Nakayama \\ Department of Applied Physics, Hokkaido University, Sapporo 060, Japan
}

(Received 4 April 1990)

\begin{abstract}
Simulations on the vibrational density of states of very large percolation clusters are presented. Above threshold $\left(p>p_{c}\right)$, not only does one find no accumulation of modes around the phononfracton crossover, but the number of modes is also depressed throughout the fracton region. These "missing modes" accumulate near the upper frequency cutoff. The reason for this pileup is explained, and scaling considerations account for the dependence on $p-p_{c}$ of the size of this effect.
\end{abstract}

The vibrational density of states (DOS) of fractal structures, initially studied by Alexander and Orbach, ' is a subject that continues to attract much attention. Fractal networks, characterized by a Hausdorff dimension $D$ and an exponent $\theta$ for the anomalous diffusion, ${ }^{2}$ have a DOS that follows the universal law $D(\omega) \propto \omega^{d-1}$, where $\tilde{d}=2 D /(2+\theta)$ is the spectral dimension. ${ }^{1,3}$ On the basis of some known values of the exponents, Alexander and Orbach conjectured that for scalar waves on infinite percolation clusters, $\tilde{d} \simeq \frac{4}{3}$, independently of the network geometry and of the underlying Euclidean dimension $d .{ }^{1}$ Such strongly localized excitations were named fractons, and $\tilde{d}$ is also called the fracton dimension.

Computer simulations have been essential to develop more insight into fracton dynamics. The early work concentrated on the existence of a phonon-fracton crossover, on the localization of fractons, and on the dependence of $\tilde{d}$ on the nature of the elastic forces. ${ }^{4,5}$ More recently, very large percolation clusters of more than $10^{5}$ particles could be investigated, revealing a wealth of detailed quantitative informations. ${ }^{6-8}$ The latter work became possible with the advent of array-processing supercomputers, and with the use of a numerical method that does not require diagonalization, as developed by Williams and Maris. ${ }^{9}$

The new simulation results confirmed that the connection between the phonon and fracton regimes is smooth, with no visible accumulation of modes, or "hump," in the DOS around the crossover frequency $\omega_{c} .{ }^{6,7,10}$ This is at variance with earlier predictions based on scaling considerations, ${ }^{11}$ as well as with calculations using the effective-medium approximation. ${ }^{12}$ The earlier considerations were based on the observation that the crossover from fractons to phonons implies "missing modes" in the normalized DOS. In the present Rapid Communication, the whereabouts of these missing modes are discussed, and we show that there are, in fact, a much larger number of them distributed over the entire fracton frequency range. We find how the weight of these modes scales with $p-p_{c}$, where $p$ is the occupation probability of the percolation network, and $p_{c}$ is the percolation threshold. All these modes tend to accumulate near the upper end of the fracton frequency range, for reasons that will be explained, and produce there a visible hump in the DOS, growing with $p-p_{c}$.

We should first recall the early considerations about missing modes. " The DOS of a percolation cluster above threshold $\left(p>p_{c}\right)$ is characterized by two regimes: the fracton DOS, $D_{\mathrm{fr}}(\omega, p) \propto \omega^{d-1}$, at high frequencies $\left(\omega>\omega_{c}\right)$, and the phonon DOS, $D_{\mathrm{ph}}(\omega, p) \propto \omega^{d-1}$, at low frequencies $\left(\omega<\omega_{c}\right)$. Assuming strict similarity in the fractal regime, one might expect $D_{\mathrm{fr}}(\omega, p)=D_{\mathrm{fr}}\left(\omega, p_{c}\right)$, where the DOS is normalized to one per particle. Since $d$ is always larger than $\tilde{d}$, it results that $D_{\text {ph }}$ is smaller than $D_{\mathrm{fr}}$ when the latter is extrapolated to phonon frequencies. As the integral of $D_{\mathrm{fr}}$ was already normalized to one for $p=p_{c}$, some modes must be missing in $D\left(\omega, p>p_{c}\right)$, in view of the existence of the phonon regime. Their spectral weight must be recovered somewhere, and it was argued that the most reasonable place for accumulation is near $\omega_{c}$, leading to a hump in the DOS, and to a corresponding hump in the low-temperature specific heat. This conclusion has influenced a large body of literature in which fracton explanations have been proposed to account for anomalies in the low-temperature specific heat of amorphous materials. The present authors already commented on this elsewhere at some length. ${ }^{6,13-15}$ The main point is that a hump is neither seen in simulations of the phononfracton crossover, ${ }^{4,6}$ nor in actual experiments on fractal materials. ${ }^{16-18}$ A separate objection is that a hump in the specific heat is not the hallmark of amorphous systems. ${ }^{13}$ Given the on-going debate on the applicability of fractons to amorphous materials, it remains of importance to clarify the question of the missing modes in the DOS of fractal networks.

In this Rapid Communication, the corresponding details of the DOS are discussed on the basis of extensive simulations. The latter, already described elsewhere,${ }^{6}$ are made on $d=2$ percolating clusters of $N$ particles of unit 
mass connected by unit springs. The equations of motion are

$$
\ddot{u}_{i}=\sum_{j} K_{i j} u_{j}
$$

where $u_{i}$ is the scalar displacement of the particle at site $i$. The forces connect nearest neighbors, with $K_{i j}=1$ if both sites $i \neq j$ are occupied, zero otherwise, and $K_{i i}=-z_{i}$, where $z_{i}$ is the number of occupied sites neighboring $i$. The DOS of this system, for $p>p_{c}$, is now written

$$
D(\omega, p)=A(p) \omega^{\tilde{d}-1} F\left(\omega / \omega_{c}\right) .
$$

For infinite site-percolation clusters, the phonon-fracton crossover frequency scales as $\omega_{c}=\Omega\left(p-p_{c}\right)^{v D / \tilde{d}}$, where $v$ is the exponent of the correlation length $\xi$, with $\xi=\Xi\left(p-p_{c}\right)^{-v}$. The scaling function $F(x)=1$ for $x \gg 1$, and $F(x)=x^{d-d}$ for $x \ll 1$. This defines $\omega_{c}$ as the abscissa of the intercept of the asymptotic phonon and fracton straight lines in a double logarithmic presentation of the DOS vs $\omega .^{19}$ For $d=2$, one has $D=\frac{91}{48}$ and $v=\frac{4}{3}$; for the underlying square lattice used here, $\Omega \simeq 13$, and $\Xi \simeq 0.95$. $^{20}$

Simulation results are illustrated in Fig. 1. They show the appropriateness of Eq. (2) in the phonon-fracton crossover region. Since one expects $\tilde{d} \simeq \frac{4}{3}$, the ordinate in Fig. 1 is the quantity $D(\omega) / \omega^{1 / 3}$ vs $\omega$, so that the fracton regime corresponds to the horizontal line of height $A(p)$. Data at the percolation threshold $\left(p_{c}=0.593\right)$ are shown by solid circles. In that case, the network was prepared on a $700 \times 700$ lattice, with $N=116991$. The AlexanderOrbach conjecture holds over the range of $\omega$ presented in the figure, without apparent crossover to a Debye regime. The reason is that, since $p=p_{c}, \xi$ is of the order of the lattice size in this simulation. With $\xi \simeq 700$, one expects $\omega_{c}=0.001$, which is below the $\omega$ range that can be shown, as insufficient accuracy is obtained at a frequency near the cutoff imposed by the lattice size. The DOS for a cluster with $p=0.67(N=317672)$ is shown with open circles. In that case, one recognizes clearly the two regimes, with

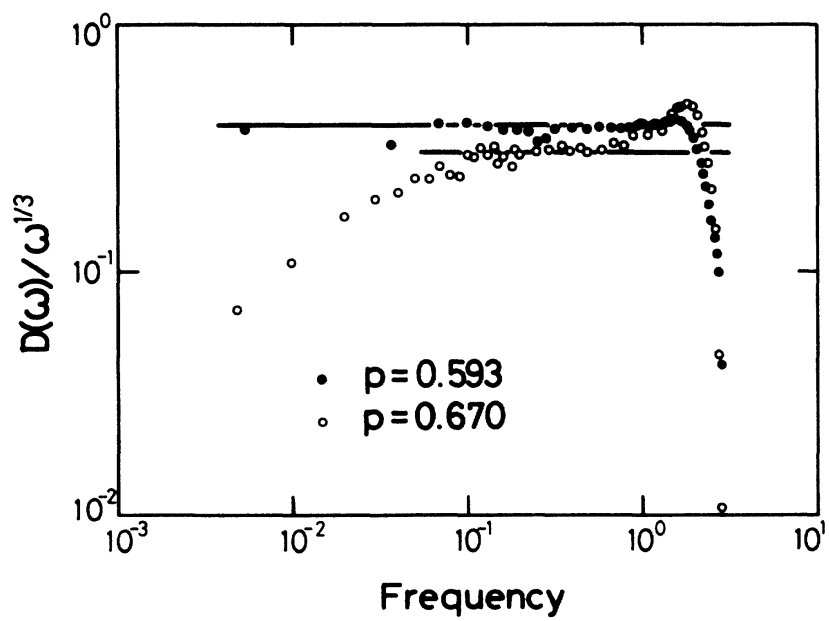

FIG. 1. The calculated DOS, normalized to one per particle, divided by $\omega^{1 / 3}$, as a function of the frequency $\omega$. The solid circles correspond to the critical percolation density $p_{c}$. The open ones are for $p=0.67$. The horizontal lines are guides to the eye. a crossover frequency $\omega_{c}=0.1$. It is clear that the simulation does not exhibit any noticeable hump near $\omega_{c}$. Further, the magnitudes of the DOS in the fracton regime are different in the two simulations, invalidating the assumption $D_{\mathrm{fr}}(\omega, p)=D_{\mathrm{fr}}\left(\omega, p_{c}\right)$, and justifying a nontrivial dependence $A(p)$ in Eq. (2). Finally, one notices that the two curves of Fig. 1 could not possibly be made to scale towards the upper end of the fracton range. This is clearly the region where modes have accumulated, invalidating Eq. (2).

To understand these observations, it is helpful to consider first the simple models illustrated in Fig. 2. From the Sierpinski gasket of Fig. 2(a), one can construct large-scale homogeneous systems in different ways. ${ }^{13}$ A first manner, illustrated in Fig. 2(b), is the carpet calculated by Southern and Douchant. ${ }^{21}$ The modes of the simple gasket, Fig. 2(a), have been investigated in considerable detail. ${ }^{22}$ They can be classified into "hierarchical" ones, whose density for $d=2$ peaks at $\omega=\sqrt{5}$, and "molecular," or strongly localized ones, with highest density near the upper cutoff, at $\omega=\sqrt{6}$. These modes are only slightly modified by the higher coordination of a few sites $(z=6)$ in Fig. 2(b). This higher $z$ just produces a few modes at frequencies above the Sierpinski gasket "band," in the region $\sqrt{6} \leq \omega \leq 3$. One notes that $\omega=3$ is the upper cutoff of the $d=2$ triangular lattice. An alternative way to construct a large-scale homogeneous system is illustrated in Fig. 2(c). That model corresponds more closely to the intuitive picture of fractal clusters that reach their correlation length by "growing into each other." In that case, the whole region $\sqrt{6} \leq \omega \leq 3$ becomes rather density populated with modes, at the expense of the DOS in the fracton regime. In fact, our preliminary simulations suggest that the relative number of modes in that high-frequency interval is very nearly equal to the relative number of sites with coordination $z=6$. The corresponding "missing" spectral weight is rather uniformly distributed over the low-frequency region.

The above considerations can now be extended to percolating clusters of finite correlation length $\xi$. The discussion is facilitated by adopting the nodes-links-blobs picture (Skal-Shklovskii-de Gennes model), ${ }^{23}$ as illustrated in Fig. 3. The backbone consists of a network of quasione-dimensional strings (links) tied together at nodes, and connecting more strongly bonded regions (blobs). The typical separation of the nodes forming the macroscopically homogeneous network equals the correlation length $\xi$. From $\xi$ upward, the network supports phonons. The vibrations of those nodes form a band that cannot extend

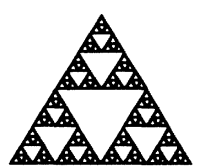

(a)

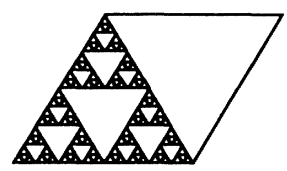

(b)

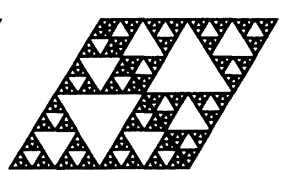

(c)
FIG. 2. Lattice models based on the Sierpinski gasket: (a) illustrates a gasket up to the fourth level of hierarchy; (b) is a unit cell made of such a gasket and an empty triangle; (c) is a denser unit cell, possibly more representative of real fractal objects, obtained by the junction of two gaskets such as in (a). 


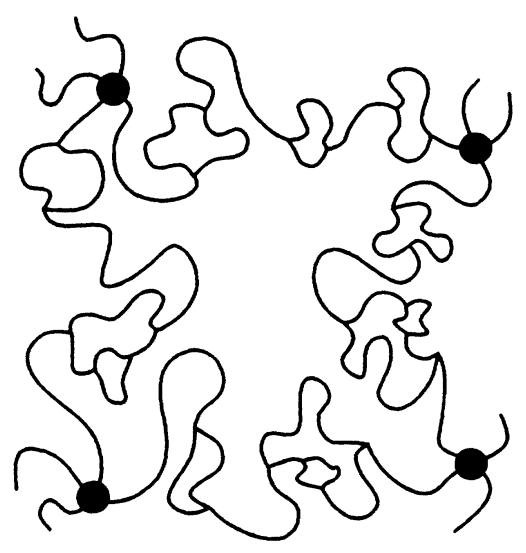

FIG. 3. Illustration of the nodes-links-blobs model for the backbone of percolation clusters. The solid circles indicate nodes forming the homogeneous network.

beyond $\omega \simeq 2 \sqrt{2}$, which is the cutoff for the square lattice. In fact, since sites near nodes are generally loaded by the larger effective mass of surrounding blobs, one expects a cutoff at a somewhat lower value. It is precisely in the region 1.5-2.0 that a strong accumulation of modes is observed (Fig. 1). With $D_{\mathrm{ph}} \simeq A(p) \omega / \omega_{c}^{2 / 3}$ and $D_{\mathrm{fr}}$ $=A(p) \omega^{1 / 3}$, one calculates the relative number of "missing modes" associated with the phonon regime $M_{\mathrm{ph}}$ as

$$
\begin{aligned}
M_{\mathrm{ph}} & =\int_{0}^{\omega_{c}}\left(D_{\mathrm{fr}}-D_{\mathrm{ph}}\right) d \omega=\frac{1}{4} A(p) \omega_{c}^{\tilde{d}} \\
& =\frac{1}{4} A(p) \Omega^{\tilde{d}}\left(p-p_{c}\right)^{v D} .
\end{aligned}
$$

With $A(p) \simeq A\left(p_{c}\right)=0.4$ from Fig. 1, one estimates $M_{\mathrm{ph}} \simeq 3\left(p-p_{c}\right)^{v D}$. This value is normalized to one per occupied site. The number of occupied sites in the correlation area $\xi^{2}$ is $\xi^{2} P_{\infty}$, where $P_{\infty}=P_{0}\left(p-p_{c}\right)^{\beta}$, with $\beta=\frac{5}{36}$ and $P_{0}=1.53 .{ }^{20}$ Hence, the actual number of missing modes within the correlation area is $\simeq \xi^{2} P_{\infty} M_{\mathrm{ph}}$ $\simeq 3 \Xi^{2} P_{0}$, which is independent of $p-p_{c}$ since the exponent $-2 v+\beta+v D$ is identically zero. Further, the actual value is of the order of one. Thus, these missing modes have approximately the weight of the phonon branch. One should also note that, for any non-negligible $\xi$, the absolute number $M_{\text {ph }}$ is very small compared to one, the corresponding area in Fig. 1 being overemphasized by the logarithmic presentation.

Numerically more important is the relative number of missing modes $\boldsymbol{M}_{\mathrm{fr}}$, produced by the depression of the fracton density from $A\left(p_{c}\right)$ to $A(p)$, as per Eq. (2). Ignoring the hump near the high-frequency cutoff, this number is

$$
M_{\mathrm{fr}}=\int_{0}^{\omega_{\max }}\left[D_{\mathrm{fr}}\left(\omega, p_{c}\right)-D_{\mathrm{fr}}(\omega, p)\right] d \omega=1-\frac{A(p)}{A\left(p_{c}\right)} .
$$

For the second equality, use was made of Eq. (2), and of the fact that the integral of $D_{\mathrm{fr}}\left(\omega, p_{c}\right)$ over the full frequency range is already normalized to one. The experimental values of $\boldsymbol{M}_{\mathrm{fr}}$ are shown in Fig. 4, demonstrating a critical behavior, $M_{\mathrm{fr}}=M_{0}\left(p-p_{c}\right)^{m}$. The solid line, drawn with $m=\frac{4}{3}$, gives $M_{0}=4.1$. The behavior can be explained tentatively as follows. Within the area $\xi^{2}$, a

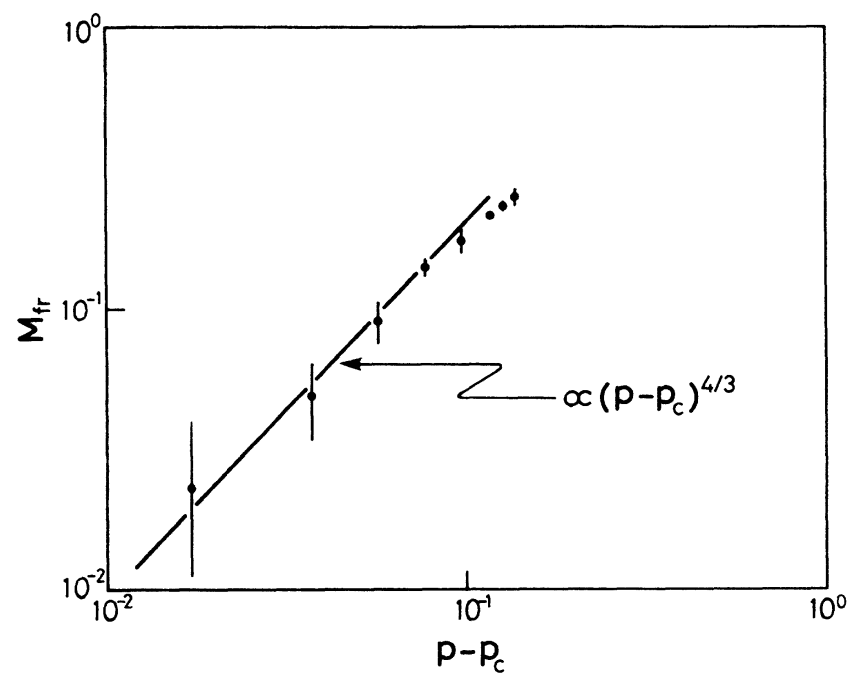

FIG. 4. The relative number of missing modes, $M_{\mathrm{fr}}$, produced by the depression in the fracton density of states, presented vs $p-p_{c}$. The error bars are standard deviations. The straight line of slope $v=\frac{4}{3}$ is shown to agree with the asymptotic behavior for $p \rightarrow p_{c}$.

number of sites have higher coordination than in the cluster at $p_{c}$. The number of these sites is much larger than the small number of nodes that eventually form the homogeneous system (Fig. 3), and whose relative density is $1 /\left(\xi^{2} P_{\infty}\right) \simeq M_{\text {ph. }}$. Based on the naive picture of Fig. $2(\mathrm{c})$, one could expect that the number of these sites is proportional to the length of the perimeter, i.e., $\propto \xi^{d-1}$. In the case of the percolation cluster, the perimeter at $\xi$ is a fractal of dimension $D-1$, and its length is $\propto \xi^{D-1}$. The total number of occupied sites within this perimeter being $\xi^{D}$, the relative number of modes rejected to high frequency is then $M_{\mathrm{fr}}=\xi^{D-1} / \xi^{D} \propto\left(p-p_{c}\right)^{v}$.

Actually, taking either a square of side $\xi$ or a circle of diameter $\xi$, the ratio of occupied perimeter sites to occupied area is exactly $4 / \xi \simeq 4.2\left(p-p_{c}\right)^{v}$, and one notices that $v=\frac{4}{3}$ for our $d=2$ case and the numerical prefactor is $\approx M_{0}$. This supports the correctness of this interpretation. Although there is, strictly speaking, no well-defined "perimeter" at $\xi$ for which the connectivity of all sites is increased, the concept appears to remain well defined from an average point of view. The effect of the higher connectivity is to depress the number of modes throughout the fracton regime.

The above considerations have been developed for the vibrational problem on Sierpinski gaskets and percolation clusters in $d=2$. One should expect that these effects are rather general, and should occur also at higher dimensions and for mode problems other than mechanical vibrations. In particular, they should be observable on superconducting deterministic fractals. Samples such as shown in Fig. 2 have actually been made by lithography, and their mode properties have been investigated. ${ }^{24}$ These seem to be rather obvious candidates for a quantitative experimental test of the above ideas.

This work was supported in part by the Foundation of the Ministry of Education, Science and Culture, Japan. 
'S. Alexander and R. Orbach, J. Phys. (Paris) Lett. 43, L625 (1982).

${ }^{2}$ Y. Gefen, A. Aharony, and S. Alexander, Phys. Rev. Lett. 50, 77 (1983).

${ }^{3}$ R. Rammal and G. Toulouse, J. Phys. (Paris) Lett. 44, L13 (1983).

${ }^{4}$ G. S. Grest and I. Webman, J. Phys. (Paris) Lett. 45, L1155 (1984).

${ }^{5}$ I. Webman and G. S. Grest, Phys. Rev. B 31, 1689 (1985).

${ }^{6}$ K. Yakubo and T. Nakayama, Phys. Rev. B 36, 8933 (1987).

${ }^{7}$ K. Yakubo and T. Nakayama, Phys. Rev. B 40, 517 (1989); J. Phys. Soc. Jpn. 58, 1504 (1989).

${ }^{8}$ T. Nakayama, K. Yakubo, and R. Orbach, J. Phys. Soc. Jpn. 58, 1891 (1989).

${ }^{9}$ M. L. Williams and H. J. Maris, Phys. Rev. B 31, 4508 (1985).

${ }^{10} \mathrm{~S}$. Russ, H. E. Roman, and A. Bunde, in Phonons 89, edited by W. Ludwig, S. Hunklinger, and G. Weiss (World Scientific, Singapore, 1989), p. 685.

"A. Aharony, S. Alexander, O. Entin-Wohlman, and R. Orbach, Phys. Rev. B 31, 2565 (1985).

${ }_{12}^{12}$ B. Derrida, R. Orbach, and K.-W. Yu, Phys. Rev. B 29, 6645 (1984).

${ }^{13}$ E. Courtens, R. Vacher, and J. Pelous, in Fractals' Physical Origin and Properties, edited by L. Pietronero (Plenum, London, 1990), p. 285.

${ }^{14}$ E. Courtens, R. Vacher, and E. Stoll, Physica D38, 41 (1989).

${ }^{15}$ T. Nakayama, in Phonons 89, (Ref. 10), p. 646.

${ }^{16}$ E. Courtens, J. Pelous, J. Phalippou, R. Vacher, and T. Woignier, Phys. Rev. Lett. 58, 128 (1987); E. Courtens, R. Vacher, J. Pelous, and T. Woignier, Europhys. Lett. 6, 245 (1988).

${ }^{17}$ E. Courtens, C. Lartigue, F. Mezei, R. Vacher, G. Coddens, M. Foret, J. Pelous, and T. Woignier, Z. Phys. B 79, 1 (1990).

${ }^{18}$ D. W. Schaefer, C. J. Brinker, D. Richter, B. Farago, and B.
Frick (unpublished).

${ }^{19}$ Other definitions of $\omega_{c}$ lead to discontinuities in $D(\omega, p)$ at $\omega_{c}$. See, for example, S. Alexander, C. Laermans, R. Orbach, and H. M. Rosenberg, Phys. Rev. B 28, 4615 (1983).

${ }^{20}$ Results on percolation are reviewed by D. Stauffer, in On Growth and Form, edited by H. E. Stanley and N. Ostrowsky (Martinus Nijhoff, Dordrecht, 1986), p. 79. The value $\Omega$ is from the present simulations. For $\Xi$, there is a certain degree of arbitrariness in the absolute definition of $\xi$, as illustrated, for example, in the work of A. Kapitulnik, A. Aharony, G. Deutcher, and D. Stauffer, J. Phys. A 16, L269 (1983), where two rather different values are found (one in their Fig. 2, and another further in the text). We use here a value of $\Xi$ derived from the mean-square size of fractons. The eigenmodes were obtained by direct diagonalization of the dynamical matrix, and their mean size is related to their frequency [E. Stoll and E. Courtens (unpublished)]. It is then assumed that the same relation holds between $\xi$ and $\omega_{c}$, which gives a value for $\Xi$ that appears most suitable for the considerations we are about to make, since the latter are intimately related to the fracton size. The value of $P_{0}$ is taken from a series analysis using Padé approximants by M. F. Sykes, D. S. Gaunt, and M. Glen, J. Phys. A 9, 725 (1976).

${ }^{21}$ B. W. Southern and A. R. Douchant, Phys. Rev. Lett. 55, 966 (1985).

${ }^{22}$ R. Rammal, J. Phys. (Paris) 45, 191 (1984).

${ }^{23}$ A. S. Skal and B. I. Shklovskii, Fiz. Tekh. Poluprov. 8, 1586 (1974) [Sov. Phys. Semicond. 8, 1029 (1975)]; P. G. de Gennes, J. Phys. (Paris) Lett. 37, Ll (1976).

${ }^{24}$ J. M. Gordon, A. M. Goldman, J. Maps, D. Costello, R. Tiberio, and B. Whitehead, Phys. Rev. Lett. 56, 2280 (1986); J. M. Gordon, A. M. Goldman, and B. Whitehead, ibid. 59, 2311 (1987). 